Research Paper

\title{
Preventable lifestyle and eating habits associated with gastric adenocarcinoma: A case-control study
}

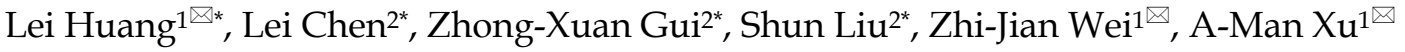 \\ 1. Department of General Surgery, the First Affiliated Hospital of Anhui Medical University; \\ 2. Second Clinical Medicine College of Anhui Medical University. \\ *Lei Huang, Lei Chen, Zhong-Xuan Gui, and Shun Liu contributed equally to this work. \\ $\bowtie$ Corresponding author: Prof. A-Man Xu (amanxu@163.com), Dr. Zhi-Jian Wei (305801533@qq.com), and Dr. Lei Huang (huangleizhenting@126.com), \\ Department of General Surgery, the First Affiliated Hospital of Anhui Medical University, 218 JiXi Avenue, Hefei 230022, Anhui, China. Phone: \\ +86-551-62922114; Fax: +86-551-63633742. \\ () The author(s). This is an open access article distributed under the terms of the Creative Commons Attribution License (https://creativecommons.org/licenses/by/4.0/). \\ See http://ivyspring.com/terms for full terms and conditions.
}

Received: 2019.08.05; Accepted: 2019.11.14; Published: 2020.01.01

\begin{abstract}
Background: Besides the well-established risk factors for gastric adenocarcinoma $(\mathrm{GaC})$, many other etiological factors remain largely unexplored. This large comprehensive case-control study aimed to investigate the preventable lifestyle and eating habits associated with $\mathrm{GaC}$.

Methods: Consecutive patients with primary microscopically-confirmed $\mathrm{GaC}$ diagnosed in 2016-2018 were matched by sex, age, height, and socioeconomic status at a 1:1 ratio with healthy controls. Association of $\mathrm{GaC}$ versus control with investigated factors was assessed using the multivariable-adjusted conditional logistic regression for paired samples.

Results: Together $302 \mathrm{GaC}$ patients and 302 healthy controls were investigated. Participants receiving higher education and those eating majorly vegetables had less frequently $\mathrm{GaC}$. The majorly frying cooking habit was associated with a higher incidence of $\mathrm{GaC}$. People complaining about poor sleep quality had more often $\mathrm{GaC}$. The more often one smoked, the more often he/she had $\mathrm{GaC}$. A higher frequency for having pickled food was associated with more frequent $\mathrm{GaC}$, while having more frequently vegetables/fruit, beans, or kelps was associated with less often $\mathrm{GaC}$. A greater preference for sour or bitter taste was associated with less frequent $\mathrm{GaC}$. The frequencies of thin liquid intake after meal, swallowing hot food without adequate cooling, doing other things while eating, eating overnight food, and eating midnight snack were all positively associated with $\mathrm{GaC}$, while going to bed regularly was associated with less often $\mathrm{GaC}$.

Conclusions: Education level, sleep quality, smoking, the frequencies of use of several foods and seasonings, the preference for specific tastes, and various eating and living habits were associated with $\mathrm{GaC}$. The findings offer important hints for further prospective investigations and for easy effective $\mathrm{GaC}$-preventative strategy-making.
\end{abstract}

Key words: Gastric adenocarcinoma; lifestyle; eating habits; etiology; case-control study.

\section{Introduction}

Gastric cancer, the majority of which is gastric adenocarcinoma $(\mathrm{GaC})$, is the $5^{\text {th }}$ most commonly diagnosed malignancy and the $3^{\text {rd }}$ leading cause of cancer mortality in both sexes combined worldwide, with $\sim 1,034,000$ new cases and $\sim 783,000$ deaths in 2018 [1]. Its incidence is highest in Eastern Asia [2]. In China, gastric cancer was estimated to affect $\sim 679,000$ patients and to cause 498,000 deaths in 2015, and was both the $2^{\text {nd }}$ most commonly diagnosed cancer and the $2^{\text {nd }}$ leading cause of cancer death in both sexes combined [3].

Helicobacter pylori $(\mathrm{Hp})$ is the major risk factor for $\mathrm{GaC}$, contributing to $\sim 90 \%$ of new cases of non-cardia $\mathrm{GaC}[4,5]$. Some unhealthy dietary habits (e.g., food 
preservation by salting and low fruit/vegetable intake), alcohol consumption, and tobacco smoking have also been shown to be associated with a higher risk of $\mathrm{GaC}[1,6-13]$. Notably, results on the associations of $\mathrm{GaC}$ with some factors (e.g., drinking, smoking, and red meat intake) remain controversial $[1,6-16]$, and many other preventable risk factors have not yet been well established.

This study aimed to comprehensively investigate the easily-modifiable lifestyle and eating habits associated with $\mathrm{GaC}$ and to offer evidence for disease prevention. Our findings can potentially aid to identify people at high risk of $\mathrm{GaC}$ and be used for risk-adapted screening.

\section{Methods}

\section{Participants}

Consecutive patients with first primary microscopically-confirmed GaC diagnosed in the First Affiliated Hospital of Anhui Medical University (FAHAMU) between July 2016 and August 2018 were included in this case-control study. Patients with previous malignancies, with cancers other than $\mathrm{GaC}$, with other benign gastric diseases, with diseases impairing memory (e.g., dementia), with severe dysfunction of important organs, or with severe systematic unfitness were excluded. They were matched by sex, age, height, and socioeconomic status at a 1:1 ratio with healthy controls confirmed not to have any gastric disorders except superficial gastritis. Since many patients with $\mathrm{GaC}$ are diagnosed at an advanced stage and are usually significantly thinner compared to the healthy controls and their pre-disease conditions, weight was not included as a matching factor. All participants did not have previous symptomatic reflux, and had fridges for food preservation. Individuals with any first-degree relative having $\mathrm{GaC}$ were excluded. Informed consents were obtained from all participants. This study was approved by the Internal Review Board of FAHAMU

\section{Collected information}

Participants were requested to carefully respond to a valid, uniform, and standardized questionnaire and to report their regular, habitual, customary, long-lasting conditions (before having obvious digestive symptoms in $\mathrm{GaC}$ patients). To ensure the validity and completeness of the responses, the completion of each questionnaire was supervised by one of the trained authors, who only explained items neutrally when necessary but did not offer any directive or indicative clues.

Information on participant characteristics (sex, age, height, weight, education level, marital status, alcohol drinking, smoking, and passive smoking) and comorbidities (hepatitis, diabetes, hypertension, and allergy) were first collected. Tumor location and differentiation were retrieved for patients. All participants were further requested to report the following: Number of people living and eating together with; eating and cooking habits; drinking water source; frequency score (FS) for intake of pork, chicken, beef, fish, processed meat, pickled food, dried food, smoked/baked food, vegetables/fruit, beans, stewed food, fried food, cereals, tuber crops, kelps, and dairy products; FS for use of yellow rice wine, soy sauce, vinegar, monosodium glutamate, chicken essence, onion/garlic, pepper, and ginger; FS for intake of thick (e.g., thick soup and milk) and thin liquid (e.g., water and juice) before, during, and after meal; FS for several eating habits (swallowing hot food without adequate cooling, not sufficiently chewing, overeating, doing other things while eating, eating deteriorated food, eating overnight food, eating within 0.5 hours after sports, eating midnight snack, and having milk before sleep); FS for eating at home, eating at canteen, and eating box lunch; FS for several sleeping habits (going to bed regularly, dreaming, and afternoon nap); FS for and time of housework and exercise per day; preference score (PS) for sour, sweet, bitter, spicy, and salty tastes; regularity score (RS) for having breakfast, lunch, and supper; degree of satiety and food intake per meal; rest hours after meal; nighttime and noontime sleeping hours; and quality of sleep.

FS was defined as: 0 , never; $1, \leq 1$ time per month; 2, 2-3 times per month; 3, 1-2 times per week; 4, 3-4 times per week; 5, 5-6 times per week; 6, 1 time per day; 7,2 times per day; 8,3 times per day; $9, \geq 4$ times per day. The frequency was modified from the Food Frequency Questionnaire [17]. PS ranged from 1 (extremely dislike) to 7 (extremely like) with an increment of 1 . RS ranged from 1 (very regular) to 5 (very irregular) with an increment of 1 .

\section{Statistical analyses}

The paired $t$ and $x^{2}$ tests were used for comparing continuous and categorical variables between groups, respectively. Associations of $\mathrm{GaC}$ versus control with the investigated factors were first computed in basic models using the multivariable conditional logistic regression for paired samples adjusting for sex, age, and height, and the significant factors were then all incorporated into a final multivariable logistic model also adjusting for sex, age, and height. Subgroup analyses were further performed for cardia and non-cardia cancers, respectively. Statistical significance was defined by 2 -sided $P<0.05$. Data analyses were performed using 
Table 2. Food and liquid intake

\begin{tabular}{|c|c|c|c|c|c|}
\hline Variable & Value/comment ${ }^{1}$ & Controls & Patients & OR $(95 \% \mathrm{CI})^{2}$ & $P_{\text {trend }}$ \\
\hline Pork & Frequency score & $4 \pm 2 ; 4(3-6)$ & $4 \pm 2 ; 4(3-6)$ & $0.89(0.81-0.98)$ & 0.019 \\
\hline Chicken & Frequency score & $3 \pm 1 ; 3(2-3)$ & $2 \pm 2 ; 2(1-3)$ & $0.95(0.85-1.07)$ & 0.401 \\
\hline Beef & Frequency score & $2 \pm 1 ; 1(1-2)$ & $1 \pm 1 ; 1(1-2)$ & $0.84(0.72-0.97)$ & 0.021 \\
\hline Fish & Frequency score & $3 \pm 1 ; 3(2-3)$ & $2 \pm 1 ; 2(1-3)$ & $0.82(0.72-0.92)$ & 0.001 \\
\hline Egg & Frequency score & $4 \pm 2 ; 5(3-6)$ & $4 \pm 2 ; 4(2-5)$ & $0.84(0.76-0.92)$ & $<0.001$ \\
\hline \multirow[t]{4}{*}{ Eggs per day } & As continuous & $1 \pm 1 ; 1(1-1)$ & $1 \pm 1 ; 1(1-1)$ & $0.74(0.55-0.98)$ & 0.037 \\
\hline & 0 & $40(13)$ & $65(23)$ & $2.17(1.38-3.40)$ & 0.003 \\
\hline & 1 & $229(76)$ & $181(63)$ & 1.00 (ref.) & \\
\hline & $\geq 2$ & $33(11)$ & $41(14)$ & $1.35(0.81-2.25)$ & \\
\hline Processed meat & Frequency score & $1 \pm 1 ; 0(0-1)$ & $1 \pm 1 ; 1(0-2)$ & $1.23(1.08-1.40)$ & 0.002 \\
\hline Pickled food & Frequency score & $3 \pm 2 ; 2(1-5)$ & $4 \pm 2 ; 4(2-6)$ & $1.21(1.12-1.31)$ & $<0.001$ \\
\hline Dried food & Frequency score & $1 \pm 1 ; 1(0-2)$ & $2 \pm 2 ; 2(1-3)$ & $1.26(1.12-1.41)$ & $<0.001$ \\
\hline Smoked/baked food & Frequency score & $1 \pm 1 ; 0(0-1)$ & $1 \pm 1 ; 0(0-1)$ & $1.19(1.02-1.38)$ & 0.028 \\
\hline Vegetables and fruit & Frequency score & $6 \pm 1 ; 6(6-7)$ & $5 \pm 2 ; 6(4-7)$ & $0.66(0.59-0.73)$ & $<0.001$ \\
\hline Beans & Frequency score & $4 \pm 2 ; 4(3-5)$ & $3 \pm 2 ; 3(2-4)$ & $0.75(0.67-0.84)$ & $<0.001$ \\
\hline Stewed food & Frequency score & $2 \pm 1 ; 1(1-2)$ & $2 \pm 1 ; 1(1-2)$ & $1.03(0.91-1.18)$ & 0.638 \\
\hline Fried food & Frequency score & $1 \pm 1 ; 1(1-2)$ & $2 \pm 1 ; 1(1-2)$ & $1.07(0.95-1.21)$ & 0.265 \\
\hline Cereals & Frequency score & $3 \pm 2 ; 3(2-4)$ & $2 \pm 2 ; 2(1-3)$ & $0.82(0.75-0.91)$ & $<0.001$ \\
\hline Tuber crops & Frequency score & $3 \pm 2 ; 3(2-4)$ & $3 \pm 2 ; 3(2-3)$ & $0.83(0.75-0.93)$ & 0.001 \\
\hline Kelps & Frequency score & $2 \pm 1 ; 2(1-3)$ & $2 \pm 1 ; 2(1-2)$ & $0.76(0.67-0.86)$ & $<0.001$ \\
\hline Yellow rice wine & Frequency score & $4 \pm 2 ; 4(2-6)$ & $3 \pm 2 ; 2(0-4)$ & $0.81(0.75-0.87)$ & $<0.001$ \\
\hline Soy sauce & Frequency score & $5 \pm 2 ; 6(4-6)$ & $5 \pm 2 ; 5(4-6)$ & $0.89(0.81-0.97)$ & 0.009 \\
\hline Vinegar & Frequency score & $4 \pm 2 ; 5(3-6)$ & $3 \pm 2 ; 3(2-6)$ & $0.84(0.78-0.91)$ & $<0.001$ \\
\hline Monosodium glutamate & Frequency score & $3 \pm 3 ; 1(0-6)$ & $3 \pm 3 ; 3(0-6)$ & $1.09(1.02-1.16)$ & 0.006 \\
\hline Chicken essence & Frequency score & $3 \pm 3 ; 2(0-6)$ & $3 \pm 3 ; 4(0-6)$ & $1.07(1.01-1.14)$ & 0.033 \\
\hline Onion and garlic & Frequency score & $6 \pm 2 ; 6(5-6)$ & $5 \pm 2 ; 5(3-7)$ & $0.81(0.74-0.89)$ & $<0.001$ \\
\hline Pepper & Frequency score & $5 \pm 2 ; 5(3-6)$ & $4 \pm 2 ; 4(2-6)$ & $0.85(0.79-0.92)$ & $<0.001$ \\
\hline Ginger & Frequency score & $5 \pm 2 ; 6(5-6)$ & $5 \pm 2 ; 5(3-6)$ & $0.79(0.72-0.86)$ & $<0.001$ \\
\hline Dairy product & Frequency score & $1 \pm 2 ; 0(0-3)$ & $1 \pm 2 ; 1(0-2)$ & $1.01(0.92-1.09)$ & 0.999 \\
\hline Sour taste & Preference score & $3 \pm 2 ; 2(2-4)$ & $3 \pm 1 ; 2(2-4)$ & $0.86(0.77-0.97)$ & 0.010 \\
\hline Sweet taste & Preference score & $4 \pm 2 ; 4(2-5)$ & $4 \pm 2 ; 4(2-5)$ & $0.91(0.82-1.01)$ & 0.077 \\
\hline Bitter taste & Preference score & $3 \pm 1 ; 2(2-4)$ & $2 \pm 1 ; 2(1-3)$ & $0.70(0.61-0.80)$ & $<0.001$ \\
\hline Spicy taste & Preference score & $4 \pm 2 ; 4(3-5)$ & $4 \pm 2 ; 4(2-5)$ & $0.82(0.74-0.91)$ & $<0.001$ \\
\hline Salty taste & Preference score & $4 \pm 1 ; 4(4-5)$ & $4 \pm 2 ; 4(3-5)$ & $0.98(0.88-1.10)$ & 0.747 \\
\hline
\end{tabular}

Categorical variables are shown as count (percentage [\%]), and continuous variables as mean \pm standard deviation.

${ }^{1}$ Frequency score assignment was as follows: 0 , never; $1, \leq 1$ time per month; 2, 2-3 times per month; 3, 1-2 times per week; 4, 3-4 times per week; 5, 5-6 times per week; 6, 1 time per day; 7, 2 times per day; 8,3 times per day; $9, \geq 4$ times per day. Preference score ranged from 1 (extremely dislike) to 7 (extremely like).

2 Odds ratio (OR) with $95 \%$ confidence interval (CI) for the association of each variable with gastric cancer versus control was calculated using multivariable logistic regression with adjustment for sex and age. Significant ORs are marked in bold. ref., reference.

Concerning eating and living habits (Table 3), the number of people living or eating together with was not significantly associated with $\mathrm{GaC}$. Compared to people having majorly vegetables for food, those keeping a balanced diet $(\mathrm{OR}=2.17)$ and having majorly meat $(\mathrm{OR}=3.77)$ were significantly more likely to have $\mathrm{GaC}$. The majorly frying cooking habit was significantly associated with a higher possibility of $\mathrm{GaC}$ compared to majorly steaming/boiling $(\mathrm{OR}=2.67)$. Drinking well water was significantly associated with $\mathrm{GaC}$ compared to tap water $(\mathrm{OR}=2.36)$. The $\mathrm{RS}$ for breakfast $(\mathrm{OR}=1.54)$, lunch $(\mathrm{OR}=1.77)$, and supper $(\mathrm{OR}=1.78)$ was significantly positively associated with $\mathrm{GaC}$, while no significant association was shown for degree of satiety. Higher FS for thin liquid intake before meal $(\mathrm{OR}=1.13)$, both thick $(\mathrm{OR}=1.13)$ and thin liquid intake during meal $(\mathrm{OR}=1.12)$, and thin liquid intake after meal $(\mathrm{OR}=1.19)$ was associated with increased odds for $\mathrm{GaC}$, while higher FS for thick liquid intake after meal was associated with less frequent $\mathrm{GaC}(\mathrm{OR}=0.89)$. The FS for overeating $(\mathrm{OR}=1.47)$, not sufficiently chewing
$(\mathrm{OR}=1.16)$, doing other things while eating $(\mathrm{OR}=1.13)$, swallowing hot food without adequate cooling $(\mathrm{OR}=1.25)$, eating deteriorated food $(\mathrm{OR}=1.87)$, eating overnight food $(\mathrm{OR}=1.16)$, eating within 0.5 hours after sports $(\mathrm{OR}=1.13)$, and eating midnight snack $(\mathrm{OR}=1.54)$ was all significantly positively associated with $\mathrm{GaC}$, while there was no significantly association between $\mathrm{GaC}$ and having milk before sleep. While eating at home was significantly associated with less frequent $\mathrm{GaC}(\mathrm{OR}=0.88$ per $1 \mathrm{FS})$, eating at canteen was significantly associated with more often $\mathrm{GaC}$ (OR=1.12 per $1 \mathrm{FS}$ ). The FS for eating box lunch was not significantly associated with GaC. No significant associations were observed for rest hours after meal, or nighttime or noontime sleep hours. Compared to good sleep quality, moderate $(\mathrm{OR}=1.88)$ and poor quality $(\mathrm{OR}=2.81)$ were associated with increased odds for $\mathrm{GaC}$. The more often one goes to bed regularly and has afternoon nap, the decreased odds for $\mathrm{GaC}(\mathrm{OR}=0.80$ and 0.93 per $1 \mathrm{FS}$, respectively). No significant associations of $\mathrm{GaC}$ with housework or exercise were observed. 
Table 3. Eating and living habits

\begin{tabular}{|c|c|c|c|c|c|}
\hline Variable & Value/comment ${ }^{1}$ & Controls & Patients & OR $(95 \% \mathrm{CI})^{2}$ & $P_{\text {trend }}$ \\
\hline No. of people living together with & As continuous & $3 \pm 2 ; 2(1-4)$ & $3 \pm 2 ; 2(1-4)$ & $1.10(1.00-1.21)$ & 0.060 \\
\hline No. of people eating together with & As continuous & $2 \pm 2 ; 2(1-4)$ & $3 \pm 2 ; 2(1-4)$ & $1.05(0.96-1.15)$ & 0.295 \\
\hline \multirow[t]{3}{*}{ Eating habit } & Majorly vegetables & $139(46)$ & $70(25)$ & 1.00 (ref.) & $<0.001$ \\
\hline & Balanced & $142(47)$ & $165(59)$ & $2.17(1.49-3.15)$ & \\
\hline & Majorly meat & $19(6)$ & $43(15)$ & $3.77(2.00-7.10)$ & \\
\hline \multirow[t]{2}{*}{ Cooking habit } & Majorly steaming/boiling & $280(93)$ & $241(83)$ & 1.00 (ref.) & $<0.001$ \\
\hline & Majorly frying & $22(7)$ & $51(17)$ & $2.67(1.55-4.59)$ & \\
\hline \multirow[t]{2}{*}{ Drinking water } & Well water & $47(16)$ & $88(30)$ & $2.36(1.57-3.55)$ & $<0.001$ \\
\hline & Tap water & $253(84)$ & $201(70)$ & 1.00 (ref.) & \\
\hline Breakfast & Regularity score & $2 \pm 1 ; 1(1-1)$ & $2 \pm 1 ; 2(1-3)$ & $1.54(1.33-1.79)$ & $<0.001$ \\
\hline Lunch & Regularity score & $1 \pm 1 ; 1(1-1)$ & $2 \pm 1 ; 2(1-3)$ & $1.77(1.48-2.11)$ & $<0.001$ \\
\hline Supper & Regularity score & $1 \pm 1 ; 1(1-1)$ & $2 \pm 1 ; 2(1-3)$ & $1.78(1.50-2.12)$ & $<0.001$ \\
\hline Degree of satiety & As continuous & $8 \pm 4$ & $8 \pm 2$ & $0.99(0.93-1.06)$ & 0.800 \\
\hline \multirow{3}{*}{ Food intake } & On diet & $42(14)$ & $30(11)$ & $0.78(0.46-1.32)$ & 0.593 \\
\hline & Normal & $195(66)$ & $182(68)$ & 1.00 (ref.) & \\
\hline & Overeating & $59(20)$ & $56(21)$ & $1.05(0.69-1.61)$ & \\
\hline Overeating & Frequency score & $0 \pm 1 ; 0(0-1)$ & $1 \pm 2 ; 0(0-2)$ & $1.47(1.28-1.68)$ & $<0.001$ \\
\hline Thick liquid intake before meal & Frequency score & $1 \pm 2 ; 0(0-1)$ & $1 \pm 2 ; 0(0-2)$ & $1.05(0.95-1.15)$ & 0.342 \\
\hline Thin liquid intake before meal & Frequency score & $1 \pm 2 ; 0(0-1)$ & $2 \pm 3 ; 1(0-3)$ & $1.13(1.05-1.21)$ & 0.001 \\
\hline Thick liquid intake during meal & Frequency score & $1 \pm 2 ; 0(0-1)$ & $2 \pm 2 ; 1(0-3)$ & $1.13(1.03-1.22)$ & 0.006 \\
\hline Thin liquid intake during meal & Frequency score & $1 \pm 2 ; 0(0-1)$ & $2 \pm 2 ; 1(0-3)$ & $1.12(1.04-1.21)$ & 0.004 \\
\hline Thick liquid intake after meal & Frequency score & $2 \pm 3 ; 1(0-5)$ & $2 \pm 2 ; 1(0-3)$ & $0.89(0.83-0.96)$ & 0.002 \\
\hline Thin liquid intake after meal & Frequency score & $1 \pm 3 ; 0(0-2)$ & $3 \pm 3 ; 2(0-5)$ & $1.19(1.11-1.27)$ & $<0.001$ \\
\hline Not sufficiently chewing & Frequency score & $2 \pm 2 ; 1(0-4)$ & $3 \pm 3 ; 2(0-5)$ & $1.16(1.08-1.23)$ & $<0.001$ \\
\hline Doing other things while eating & Frequency score & $1 \pm 2 ; 0(0-1)$ & $2 \pm 2 ; 0(0-2)$ & $1.13(1.04-1.22)$ & 0.005 \\
\hline Swallowing hot food without adequate cooling & Frequency score & $1 \pm 2 ; 0(0-2)$ & $3 \pm 3 ; 2(0-5)$ & $1.25(1.16-1.35)$ & $<0.001$ \\
\hline Eating deteriorated food & Frequency score & $0 \pm 1 ; 0(0-0)$ & $1 \pm 1 ; 0(0-1)$ & $1.87(1.48-2.35)$ & $<0.001$ \\
\hline Eating overnight food & Frequency score & $2 \pm 2 ; 2(0-4)$ & $3 \pm 3 ; 3(1-5)$ & $1.16(1.06-1.26)$ & 0.001 \\
\hline Eating within $0.5 \mathrm{~h}$ after sports & Frequency score & $1 \pm 2 ; 0(0-2)$ & $2 \pm 2 ; 1(0-3)$ & $1.13(1.03-1.23)$ & 0.008 \\
\hline Rest hours after meal & As continuous & $1 \pm 1 ; 1(0-1)$ & $1 \pm 1 ; 1(1-1)$ & $1.05(0.82-1.34)$ & 0.723 \\
\hline Eating midnight snack & Frequency score & $0 \pm 1 ; 0(0-0)$ & $1 \pm 2 ; 0(0-1)$ & $1.54(1.29-1.83)$ & $<0.001$ \\
\hline Eating at home & Frequency score & $7 \pm 2 ; 8(7-8)$ & $7 \pm 2 ; 8(6-8)$ & $0.88(0.81-0.96)$ & 0.004 \\
\hline Eating at canteen & Frequency score & $1 \pm 2 ; 0(0-0)$ & $1 \pm 2 ; 0(0-1)$ & $1.12(1.02-1.22)$ & 0.017 \\
\hline Eating box lunch & Frequency score & $1 \pm 2 ; 0(0-1)$ & $1 \pm 2 ; 1(0-2)$ & $1.06(0.96-1.17)$ & 0.256 \\
\hline Milk before sleep & Frequency score & $1 \pm 2 ; 0(0-1)$ & $1 \pm 1 ; 0(0-1)$ & $0.93(0.84-1.03)$ & 0.183 \\
\hline Nighttime sleep hours & As continuous & $8 \pm 1 ; 8(7-8)$ & $8 \pm 1 ; 8(7-8)$ & $0.94(0.84-1.06)$ & 0.341 \\
\hline Noontime sleep hours & As continuous & $1 \pm 1 ; 1(1-2)$ & $1 \pm 1 ; 1(0-2)$ & $0.99(0.81-1.23)$ & 0.957 \\
\hline \multirow[t]{3}{*}{ Sleep quality } & Good & $94(31)$ & $46(17)$ & 1.00 (ref.) & $<0.001$ \\
\hline & Moderate & $90(30)$ & $82(31)$ & $1.88(1.17-3.02)$ & \\
\hline & Poor & $115(38)$ & $140(52)$ & $2.81(1.80-4.39)$ & \\
\hline Going to bed regularly & Frequency score & $5 \pm 2 ; 6(4-6)$ & $4 \pm 2 ; 4(1-6)$ & $0.80(0.74-0.87)$ & $<0.001$ \\
\hline Dreaming & Frequency score & $3 \pm 2 ; 3(1-5)$ & $3 \pm 2 ; 3(2-5)$ & $1.02(0.94-1.11)$ & 0.608 \\
\hline Afternoon nap & Frequency score & $4 \pm 2 ; 4(1-6)$ & $3 \pm 2 ; 4(1-6)$ & $0.93(0.87-1.00)$ & 0.048 \\
\hline Heavy housework & Frequency score & $3 \pm 3 ; 3(1-6)$ & $3 \pm 3 ; 2(1-6)$ & $1.00(0.94-1.07)$ & 0.925 \\
\hline Light housework & Frequency score & $5 \pm 2 ; 5(3-6)$ & $4 \pm 3 ; 5(2-6)$ & $1.01(0.94-1.09)$ & 0.724 \\
\hline Housework hours per day & As continuous & $2 \pm 2 ; 2(1-4)$ & $3 \pm 2 ; 2(1-3)$ & $1.06(0.98-1.15)$ & 0.158 \\
\hline Exercise & Frequency score & $3 \pm 3 ; 3(0-6)$ & $3 \pm 3 ; 3(0-5)$ & $0.95(0.89-1.01)$ & 0.081 \\
\hline Exercise hours per day & As continuous & $2 \pm 3 ; 1(0-3)$ & $2 \pm 3 ; 1(0-3)$ & $1.02(0.96-1.09)$ & 0.491 \\
\hline
\end{tabular}

Categorical variables are shown as count (percentage [\%]), and continuous variables as mean \pm standard deviation.

${ }^{1}$ Regularity score ranged from 1 (very regular) to 5 (very irregular). Frequency score assignment was as follows: 0 , never; $1, \leq 1$ time per month; $2,2-3$ times per month; $3,1-2$ times per week; 4, 3-4 times per week; 5, 5-6 times per week; 6,1 time per day; 7,2 times per day; 8,3 times per day; $9, \geq 4$ times per day.

${ }^{2}$ Odds ratio (OR) with $95 \%$ confidence interval (CI) for the association of each variable with gastric cancer versus control was calculated using multivariable logistic regression with adjustment for sex and age. Significant ORs are marked in bold. ref., reference.

\section{Final multivariable model}

In the final multivariable model (Table 4), participants receiving primary school $(\mathrm{OR}=0.27)$ or middle school education (OR=0.21) had significantly less often $\mathrm{GaC}$ compared to those uneducated. Compared to people keeping a balanced diet, those having majorly vegetables had significantly less frequently $\mathrm{GaC} \quad(\mathrm{OR}=0.23)$. The majorly frying cooking habit was significantly associated with a higher incidence of $\mathrm{GaC}$ compared to the majorly steaming/boiling habit $(\mathrm{OR}=10.23)$. Compared to people having good sleep quality, those complaining about poor sleep quality had significantly more often $\mathrm{GaC}(\mathrm{OR}=3.18)$. The more often one smoked, the more often he/she had GaC (OR=1.28 per 1 FS). Higher FS for having pickled food was significantly associated with more frequent $\mathrm{GaC}(\mathrm{OR}=1.41)$, while having more frequently vegetables/fruit $(\mathrm{OR}=0.60)$, beans $(\mathrm{OR}=0.73)$, or kelps $(\mathrm{OR}=0.72)$ was significantly associated with less often $\mathrm{GaC}$. A greater PS for sour $(\mathrm{OR}=0.77)$ or bitter taste $(\mathrm{OR}=0.50)$ was significantly associated with less frequent $\mathrm{GaC}$. The FS for thin liquid intake after meal ( $\mathrm{OR}=1.27)$, swallowing hot food without adequate cooling $(\mathrm{OR}=1.21)$, doing other 
things while eating $(\mathrm{OR}=1.23)$, eating overnight food $(\mathrm{OR}=1.25)$, and eating midnight snack $(\mathrm{OR}=1.49)$ was all significantly positively associated with $\mathrm{GaC}$, while going to bed regularly was significantly associated with less often $\mathrm{GaC}(\mathrm{OR}=0.81$ per $1 \mathrm{FS})$.

The association patterns for cardia and non-cardia cancers were mostly similar with the whole cases, only with few exceptions. For cardia cancers, The FS for having eggs was significantly associated with less often $\mathrm{GaC}(\mathrm{OR}=0.32)$. The FS for eating vegetables/fruit was more strongly associated with reduced cardia carcinoma frequency $(\mathrm{OR}=0.16)$ compared to overall and non-cardia carcinomas. The FS for vinegar use $(\mathrm{OR}=0.65)$ and the PS for spicy taste $(\mathrm{OR}=0.57)$ were only significantly negatively associated with non-cardia cancers. More frequent pepper use was only significantly associated with less often cardia cancers $(\mathrm{OR}=0.47)$, and more often thin liquid intake during meal $(\mathrm{OR}=2.27)$, more often chewing insufficiently $(\mathrm{OR}=2.69)$, more frequently eating deteriorated food $(\mathrm{OR}=7.84)$, and more irregular supper intake $(\mathrm{OR}=6.37)$ were only significantly associated with more frequent cardia cancers.

\section{Discussion}

This study comprehensively reported eating and living habits associated with $\mathrm{GaC}$ in a large Chinese population, offering further insights into potentially modifiable factors and providing important evidence for making GaC-preventive strategies. Furthermore, some differences in association patterns and/or strengths between cardia and non-cardia cancers were found for some factors.

We found that people receiving primary or middle school education had significantly less often $\mathrm{GaC}$ compared to uneducated people, which is consistent with some previous studies showing that better education was associated with reduced $\mathrm{GaC}$ risk $[18,19]$.

Table 4. Factors associated with gastric cancer using full multivariable-adjusted model, overall and by location

\begin{tabular}{|c|c|c|c|c|c|c|c|}
\hline Variable & Value/comment ${ }^{1}$ & Overall gastric car & & Cardia cancer & & Non-cardia cancer & \\
\hline & & OR $(95 \% \mathrm{CI})^{2}$ & $P_{\text {trend }}$ & OR $(95 \% \mathrm{CI})^{2}$ & $P_{\text {trend }}$ & OR $(95 \% \mathrm{CI})^{2}$ & $P_{\text {trend }}$ \\
\hline \multirow[t]{5}{*}{ Education } & Uneducated & 1.00 (ref.) & 0.014 & 1.00 (ref.) & 0.079 & 1.00 (ref.) & 0.012 \\
\hline & Primary school & $0.27(0.09-0.78)$ & & $0.03(<0.01-0.83)$ & & $0.11(0.02-0.64)$ & \\
\hline & Middle school & $0.21(0.06-0.72)$ & & $<0.01(<0.01-0.12)$ & & $0.21(0.03-1.34)$ & \\
\hline & High school & $1.51(0.38-6.03)$ & & $0.09(<0.01-4.27)$ & & $1.81(0.24-14.00)$ & \\
\hline & College/university & $0.46(0.10-2.12)$ & & $0.20(<0.01-15.73)$ & & $0.05(<0.01-0.66)$ & \\
\hline \multirow[t]{3}{*}{ Eating habit } & Majorly vegetables & $0.23(0.09-0.56)$ & 0.003 & $0.03(<0.01-0.44)$ & 0.037 & $0.11(0.02-0.46)$ & 0.011 \\
\hline & Balanced & 1.00 (ref.) & & 1.00 (ref.) & & 1.00 (ref.) & \\
\hline & Majorly meat & $0.34(0.10-1.19)$ & & $0.32(0.01-9.57)$ & & $0.43(0.07-2.62)$ & \\
\hline \multirow[t]{2}{*}{ Cooking habit } & Majorly steaming/boiling & 1.00 (ref.) & 0.001 & & & & \\
\hline & Majorly frying & $10.23(2.70-38.80)$ & & & & & \\
\hline \multirow[t]{3}{*}{ Sleep quality } & Good & 1.00 (ref.) & 0.015 & & & & \\
\hline & Moderate & $1.01(0.38-2.70)$ & & & & & \\
\hline & Poor & $3.18(1.23-8.23)$ & & & & & \\
\hline Smoking & Frequency score & $1.28(1.12-1.46)$ & $<0.001$ & $1.64(1.12-2.41)$ & 0.012 & $1.32(1.07-1.61)$ & 0.009 \\
\hline Egg & Frequency score & & & $0.32(0.12-0.83)$ & 0.019 & & \\
\hline Pickled food & Frequency score & 1.41 (1.16-1.71) & $<0.001$ & $2.00(1.10-3.63)$ & 0.023 & $1.55(1.12-2.14)$ & 0.008 \\
\hline Vegetables and fruit & Frequency score & $0.60(0.46-0.79)$ & $<0.001$ & $0.16(0.06-0.45)$ & 0.001 & $0.73(0.51-1.05)$ & 0.094 \\
\hline Beans & Frequency score & $0.73(0.55-0.97)$ & 0.032 & & & $0.54(0.35-0.86)$ & 0.009 \\
\hline Kelps & Frequency score & $0.72(0.54-0.96)$ & 0.026 & $0.24(0.09-0.61)$ & 0.003 & & \\
\hline Vinegar & Frequency score & & & & & $0.65(0.46-0.92)$ & 0.015 \\
\hline Pepper & Frequency score & $0.84(0.68-1.03)$ & 0.090 & $0.47(0.26-0.83)$ & 0.010 & & \\
\hline Sour taste & Preference score & $0.77(0.59-1.00)$ & 0.047 & & & & \\
\hline Bitter taste & Preference score & $0.50(0.36-0.69)$ & $<0.001$ & $0.14(0.05-0.41)$ & $<0.001$ & $0.38(0.22-0.65)$ & 0.001 \\
\hline Spicy taste & Preference score & & & & & $0.57(0.38-0.86)$ & 0.008 \\
\hline Thin liquid intake during meal & Frequency score & & & $2.27(1.12-4.61)$ & 0.024 & & \\
\hline Thin liquid intake after meal & Frequency score & $1.27(1.06-1.52)$ & 0.008 & & & $1.45(1.12-1.87)$ & 0.005 \\
\hline Swallowing hot food without adequate cooling & Frequency score & $1.21(1.00-1.47)$ & 0.045 & & & $1.44(1.07-1.95)$ & 0.017 \\
\hline Supper & Regularity score & & & $6.37(1.20-33.88)$ & 0.030 & & \\
\hline Not sufficiently chewing & Frequency score & $1.18(1.00-1.40)$ & 0.058 & $2.69(1.48-4.86)$ & 0.001 & & \\
\hline Doing other things while eating & Frequency score & $1.23(1.01-1.50)$ & 0.041 & & & $1.50(1.10-2.05)$ & 0.011 \\
\hline Eating deteriorated food & Frequency score & $1.37(0.95-1.96)$ & 0.093 & $7.84(2.32-26.49)$ & 0.001 & & \\
\hline Eating overnight food & Frequency score & $1.25(1.03-1.52)$ & 0.025 & & & $1.57(1.13-2.18)$ & 0.007 \\
\hline Eating midnight snack & Frequency score & $1.49(1.09-2.03)$ & 0.013 & $3.57(1.04-12.30)$ & 0.044 & $2.04(1.22-3.41)$ & 0.007 \\
\hline Going to bed regularly & Frequency score & $0.81(0.68-0.96)$ & 0.018 & & & $0.74(0.56-0.96)$ & 0.025 \\
\hline \multicolumn{8}{|c|}{$\begin{array}{l}\text { 1Frequency score assignment was as follows: } 0 \text {, never; } 1, \leq 1 \text { time per month; } 2,2-3 \text { times per month; } 3,1-2 \text { times per week; } 4,3-4 \text { times per week; } 5,5-6 \text { times per week; } 6,1 \\
\text { time per day; } 7,2 \text { times per day; } 8,3 \text { times per day; } 9, \geq 4 \text { times per day. Preference score ranged from } 1 \text { (extremely dislike) to } 7 \text { (extremely like). Regularity score ranged fron } \\
1 \text { (very regular) to } 5 \text { (very irregular). }\end{array}$} \\
\hline
\end{tabular}


Better education could help to form and keep healthy eating and living habits, while well-educated people might face greater pressure in this competitive modern era. The associations for those receiving high school and college/university education were insignificant in overall patients and most subgroups, which could be partly explained by the small case numbers in these groups. People having majorly vegetables for food had significantly less frequent $\mathrm{GaC}$, which is also supported by previous studies [20, 21]. Furthermore, we found that more frequent vegetable/fruit intake was significantly associated with a reduced frequency of cardia cancer but not of non-cardia cancer. Previous evidence remains controversial regarding the association between red meat intake and $\mathrm{GaC}$ risk [16], and The Netherlands Cohort Study did not show a significant association [15]. The insignificance for the habit of majorly meat intake in our study might be partly explained by the paucity of participants in that group. The majorly frying cooking habit, which could generate various carcinogenic substances in a temperature-dependent manner, was associated with a higher overall $\mathrm{GaC}$ incidence compared to majorly steaming/boiling. We found that poor sleep quality was significantly associated with a higher $\mathrm{GaC}$ incidence compared to good quality. Notably, sleep quality could be influenced by various factors like time to go to bed and psychiatric status. We further found that the frequency of going to bed regularly was significantly associated with a reduced risk of $\mathrm{GaC}$ especially non-cardia cancer. Our finding that smoking was associated with $\mathrm{GaC}$ in a dose-dependent manner was well supported by previous literature [22]; however, we did not observe a significant association for alcohol drinking frequency, on which previous evidence remains controversial [14].

The frequency of pickled food intake, a well-recognized risk factor for $\mathrm{GaC}$ [23], was associated with increased risks of both cardia and non-cardia cancers. Interestingly, more frequent egg intake was significantly associated with a reduced risk of cardia cancer but not of non-cardia cancer. While higher frequencies of intake of beans and kelps were both significantly associated with a decreased overall $\mathrm{GaC}$ risk, beans intake was significantly associated with non-cardia cancer and kelps intake with cardia cancer. More often vinegar use was significantly associated with a reduced non-cardia cancer risk, and a greater preference for sour taste was significantly associated with a lower overall GaC risk. We previously reported that distal $\mathrm{GaC}$ was mostly associated with hypoacidity [24], and adequate acidification of inner-stomach environment might be protective against malignancy, possibly by inhibiting growth and proliferation of organisms. More frequent pepper use was associated with a lower incidence of cardia cancer, and a greater preference of spicy taste was significantly associated with a lower cardia cancer risk. However, an early study [25] reported that Chili pepper consumption was positively associated with $\mathrm{GaC}$ risk. The discrepancies from our findings could be possibly due to the different strains between Asia and South America. Notably, a greater preference for bitter taste was significantly associated with reduced incidences of both cardia and non-cardia cancers. The associations with various food and seasoning intake and flavor preference offer important clues for easy GaC-preventative strategy making, which should be further validated by prospective studies.

Some specific eating habits were further found to be associated with $\mathrm{GaC}$ risk through multivariable analysis. The more often one had thin liquid during meal, the more probably he/she had cardia adenocarcinoma, while more frequent thin liquid intake after meal was significantly associated with an increased risk of non-cardia cancer. Thin liquid intake during/after meal could dilute the gastric liquid, thus increasing the burden of stomach. Notably, we did not observe a significant association for thick liquid intake. Swallowing hot food without adequate cooling was associated with an increased $\mathrm{GaC}$ risk, which might be due to the damaging effect of heat to gastric mucosa. Insufficiently chewing was associated with an increased $\mathrm{GaC}$ risk, which could be attributed to the increased digestive burden for the stomach. Doing other things while eating could reduce the blood flow to the stomach, potentially causing the organ to be more vulnerable. The frequencies of eating deteriorated and overnight food, which might contain increased carcinogenic microorganisms and chemical compounds, were both associated with an increased $\mathrm{GaC}$ risk. Among 3 meals in a day, only the irregularity degree of supper was significantly associated with cardia cancer. A short interval between having supper and going to bed could induce and accelerate reflux, a risk factor for cardia cancer. Accordingly, eating midnight snack was significantly associated with an increased $\mathrm{GaC}$ risk. Nearly all of these potential $\mathrm{GaC}$ risk factors could be modifiable. If prospectively validated, GaCpreventative strategies could be accordingly made.

This case-control study was limited by its retrospective observational nature. The associations observed do not suggest causality, and should be validated in prospective cohorts. Recall bias could affect the accuracy of the results. There could be other risk factors that have not been accounted for in this study (e.g., depression). Hp infection status was not 
adjusted for in this study, considering that the measure for cancer patients might not reflect the real pre-cancer status. Some originally $\mathrm{Hp}$-infected patients may have the infection status turn negative during the development of cancer. Furthermore, it would be difficult to know the exact duration of infection which might differ largely between the patient and control groups. The case numbers in some subgroups were not large enough to obtain statistical significance, and larger relevant studies are encouraged. Notably, the risk factors for $\mathrm{GaC}$ in Western people could be different from those in Asian people. Molecular and genetic risk factors could potentially further help to identify people at risk.

Our study is a large comprehensive investigation on various easily modifiable factors potentially causing $\mathrm{GaC}$ in Asian people. Further subgroup analyses according to tumor location were conducted. While some identified $\mathrm{GaC}$-associated factors have been reported previously, there are various newly detected modifiable and preventable eating and living habits, which provide important informative clues for future investigations and which will contribute greatly to $\mathrm{GaC}$ prevention if validated prospectively. Once validated, the findings can serve as references for making effective population-based strategies to prevent $\mathrm{GaC}$. Through health education campaigns to raise the public awareness of the modifiable and preventable factors associated with $\mathrm{GaC}$, it can be expected that a significant proportion of $\mathrm{GaC}$ cases can be avoided in a cost-effective manner, especially for individuals without $\mathrm{Hp}$ infection who may have poorer prognosis if developing $\mathrm{GaC}$ [26]. Our evidence-based findings provide novel clues to help to identify people at a high risk of $\mathrm{GaC}$ which can be potentially used for risk-adapted screening and which may contribute to early diagnosis. Modifying the validated factors may even help to prolong survival and improve quality of life for patients with $\mathrm{GaC}$, and further studies in these aspects are needed.

In conclusion, education level, sleep quality, smoking, the frequencies of use of several foods and seasonings, the preference for specific tastes, and various eating and living habits were significantly associated with $\mathrm{GaC}$, with some location-specific differences. Our findings offer important hints for further prospective investigations and for easy effective $\mathrm{GaC}$-preventative strategy making.

\section{Acknowledgements}

The authors would most sincerely thank the reviewers and editors for critically reviewing this paper and for the constructive and thoughtful comments and suggestions.

\section{Funding}

This work was supported by National College Students' Innovation and Entrepreneurship Training Program (9021446101) and National Natural Science Foundation of China (81572350). The funders had no role in study design, data collection and analysis, decision to publish, or preparation of the manuscript.

\section{Authors' contributions}

Huang L, Chen L, Gui ZX, Liu S, Wei ZJ, and Xu AM designed the research; Huang L, Chen L, Gui ZX, Liu S, and Wei ZJ performed the research; Huang L analyzed and interpreted the data, and wrote the manuscript; Chen L, Gui ZX, Liu S, Wei ZJ, and Xu AM critically reviewed the paper.

\section{Ethics approval and consent to participate}

This study was approved by the Institutional Review Board of First Affiliated Hospital of Anhui Medical University. Written informed consent was obtained from each investigated individual. No individual patient data were reported.

\section{Availability of data and materials}

The data that support the findings of this study are available from our center but restrictions apply to the availability of these data, which were used under license for the current study, and so are not publicly available.

\section{Grant support}

This work was supported by National College Students' Innovation and Entrepreneurship Training Program (9021446101) and National Natural Science Foundation of China (81572350). The funders had no role in study design, data collection and analysis, decision to publish, or preparation of the manuscript.

\section{Competing Interests}

The authors have declared that no competing interest exists.

\section{References}

1. Bray F, Ferlay J, Soerjomataram I, Siegel RL, Torre LA, Jemal A. Global cancer statistics 2018: GLOBOCAN estimates of incidence and mortality worldwide for 36 cancers in 185 countries. CA Cancer J Clin. 2018; 68: 394-424.

2. Huang $\mathrm{L}, \mathrm{Xu} \mathrm{AM}$. Adenocarcinoma of esophagogastric junction: controversial classification, surgical management, and clinicopathology. Chin J Cancer Res. 2014; 26: 226-30.

3. Chen $\mathrm{W}$, Zheng $\mathrm{R}$, Baade PD, Zhang $\mathrm{S}$, Zeng $\mathrm{H}$, Bray $\mathrm{F}$, et al. Cancer statistics in China, 2015. CA Cancer J Clin. 2016; 66: 115-32.

4. Plummer M, Franceschi S, Vignat J, Forman D, de Martel C. Global burden of gastric cancer attributable to Helicobacter pylori. Int J Cancer. 2015; 136: 487-90.

5. Islami F, Chen W, Yu XQ, Lortet-Tieulent J, Zheng R, Flanders WD, et al. Cancer deaths and cases attributable to lifestyle factors and infections in China, 2013. Ann Oncol. 2017; 28: 2567-74.

6. Wang Z, Koh WP, Jin A, Wang R, Yuan JM. Composite protective lifestyle factors and risk of developing gastric adenocarcinoma: the Singapore Chinese Health Study. Br J Cancer. 2017; 116: 679-87. 
7. Buckland G, Travier N, Huerta JM, Bueno-de-Mesquita HB, Siersema PD, Skeie $G$, et al. Healthy lifestyle index and risk of gastric adenocarcinoma in the EPIC cohort study. Int J Cancer. 2015; 137: 598-606.

8. Navarro Silvera SA, Mayne ST, Gammon MD, Vaughan TL, Chow WH, Dubin JA, et al. Diet and lifestyle factors and risk of subtypes of esophageal and gastric cancers: classification tree analysis. Ann Epidemiol. 2014; 24: 50-7.

9. Lee YY, Derakhshan MH. Environmental and lifestyle risk factors of gastric cancer. Arch Iran Med. 2013; 16: 358-65.

10. Navarro Silvera SA, Mayne ST, Risch HA, Gammon MD, Vaughan T, Chow $\mathrm{WH}$, et al. Principal component analysis of dietary and lifestyle patterns in relation to risk of subtypes of esophageal and gastric cancer. Ann Epidemiol. 2011; $21: 543-50$

11. Kim RH, Chang MS, Kim HJ, Song KS, Kim YS, Choi BY, et al. Medical history and lifestyle factors contributing to Epstein-Barr virus-associated gastric carcinoma and conventional gastric carcinoma in Korea. Anticancer Res. 2010; 30: 2469-75.

12. Yamaji $\mathrm{Y}$, Watabe $\mathrm{H}$, Yoshida $\mathrm{H}$, Kawabe $\mathrm{T}$, Wada $\mathrm{R}$, Mitsushima $\mathrm{T}$, et al. High-risk population for gastric cancer development based on serum pepsinogen status and lifestyle factors. Helicobacter. 2009; 14: 81-6.

13. Chen MJ, Chiou YY, Wu DC, Wu SL. Lifestyle habits and gastric cancer in a hospital-based case-control study in Taiwan. Am J Gastroenterol. 2000; 95: 3242-9.

14. Tramacere I, Negri E, Pelucchi C, Bagnardi V, Rota M, Scotti L, et al. A meta-analysis on alcohol drinking and gastric cancer risk. Ann Oncol. 2012; 23: $28-36$

15. Keszei AP, Schouten LJ, Goldbohm RA, van den Brandt PA. Red and processed meat consumption and the risk of esophageal and gastric cancer subtypes in The Netherlands Cohort Study. Ann Oncol. 2012; 23: 2319-26.

16. Song P, Lu M, Yin Q, Wu L, Zhang D, Fu B, et al. Red meat consumption and stomach cancer risk: a meta-analysis. J Cancer Res Clin Oncol. 2014; 140: 979-92.

17. Subar AF, Thompson FE, Kipnis V, Midthune D, Hurwitz P, McNutt S, et al. Comparative validation of the Block, Willett, and National Cancer Institute food frequency questionnaires : the Eating at America's Table Study. Am J Epidemiol. 2001; 154: 1089-99.

18. Lagergren J, Andersson G, Talback M, Drefahl S, Bihagen E, Harkonen J, et al. Marital status, education, and income in relation to the risk of esophageal and gastric cancer by histological type and site. Cancer. 2016; 122: 207-12

19. Trujillo Rivera A, Sampieri CL, Morales Romero J, Montero H, Acosta Mesa HG, Cruz Ramirez N, et al. Risk factors associated with gastric cancer in Mexico: education, breakfast and chili. Rev Esp Enferm Dig. 2018; 110: 372-9.

20. Wang T, Cai H, Sasazuki S, Tsugane S, Zheng W, Cho ER, et al. Fruit and vegetable consumption, Helicobacter pylori antibodies, and gastric cancer risk: A pooled analysis of prospective studies in China, Japan, and Korea. Int J Cancer. 2017; 140: 591-9.

21. Peleteiro B, Padrao P, Castro C, Ferro A, Morais S, Lunet N. Worldwide burden of gastric cancer in 2012 that could have been prevented by increasing fruit and vegetable intake and predictions for 2025. Br J Nutr. 2016; 115: 851-9.

22. Nomura AM, Wilkens LR, Henderson BE, Epplein M, Kolonel LN. The association of cigarette smoking with gastric cancer: the multiethnic cohort study. Cancer Causes Control. 2012; 23: 51-8.

23. Ren JS, Kamangar F, Forman D, Islami F. Pickled food and risk of gastric cancer--a systematic review and meta-analysis of English and Chinese literature. Cancer Epidemiol Biomarkers Prev. 2012; 21: 905-15.

24. Huang L, Xu AM, Li TJ, Han WX, Xu J. Should peri-gastrectomy gastric acidity be our focus among gastric cancer patients? World J Gastroenterol. 2014; 20: 6981-8.

25. Lopez-Carrillo L, Hernandez Avila M, Dubrow R. Chili pepper consumption and gastric cancer in Mexico: a case-control study. Am J Epidemiol. 1994; 139: 263-71.

26. Marrelli D, Pedrazzani C, Berardi A, Corso G, Neri A, Garosi L, et al. Negative Helicobacter pylori status is associated with poor prognosis in patients with gastric cancer. Cancer. 2009; 115: 2071-80. 